\title{
Random Channel Generator for Indoor Power Line Communication
}

\author{
P. Mlynek, J. Misurec, M. Koutny \\ Department of Telecommunications, Faculty of Electrical Engineering and Communication, Brno University of Technology, \\ Technicka 12, 61200 Brno, Czech Republic, mlynek@feec.vutbr.cz, misurec@feec.vutbr.cz, koutnym@feec.vutbr.cz

\begin{abstract}
The paper deals with creating an indoor power line model based on random parameters. This model approximates the real parameters of the power line communication with sufficient precision. A detailed analysis of earlier and current research in power line communication modelling, especially for power line models, is described. Measurement of transmission line parameters and power line model verification follows. Based on model analysis and load impedance measurement, a mathematical description of the model is designed. A reference model for different scenarios is realized too. The last part gives the analysis of this model and simulation results.
\end{abstract}

Keywords: Power line communication, linear time-invariant system, measurement, model, generator, transfer function.

\section{INTRODUCTION}

$\mathrm{S}$ YSTEMS FOR COMMUNICATION over power lines are referred to as Power Line Communication (PLC). Nowadays, power lines are considered as an alternative transmission channel mainly due to economic advantages.

The main use of the PLC technology these days can be divided into two categories:

- Broadband PLC is a suitable communication channel inside buildings (indoor power line) for Smart Grid networks [1] and Smart Home[2].

- Narrowband PLC seems an appropriate communication channel for remote data acquisition, automatic meter readings (AMR systems [3]) or remote fault localization and fault type recognition [4],[5].

These arguments are supported by an increasing interest in exploring this alternative communication channel [6]-[8].

Recently, it has been shown that PLC systems can be used to provide high-speed data rates by using a bandwidth of up to $100 \mathrm{MHz}$ as defined in the G.hn standard [9].

On the other hand, there are still several problematic areas in data transmission over power lines. The power line channels differ considerably in topology, structure and physical properties from conventional media such as twisted pair or coaxial cables [10]. The topology of the network, the length of lines, and appliances connected to the terminal nodes can be determined at random. Randomizing these parameters allows studying the channel sensitivity to physical characteristic of the network.

Power line channel is a very harsh and noisy transmission medium [11]. The power line channel is frequency-selective, time-varying, and is impaired by noise. Therefore, it is very difficult to model [12].

Modelling the power line can be aided by analysing the multipath nature of the power grid, arising from the presence of several branches and impedance mismatches.

The key to the Smart Grid vision is the communications network, which serves as the fundamental information infrastructure to provide bidirectional communication in the Smart Grid. Existing communication technologies can be applied to the Smart Grid, new communication protocol and enhancement of the existing protocol are necessary to capture the unique characteristic and requirement of Smart Grid. Sufficiently precise computer models of PLC systems will make the process of selecting and deploying new telemetry and management technologies for Smart Grids markedly more effective [13]-[15].

\section{POWER LINE MODELLING FUNDAMENTALS}

The most widely used model for the power line channel transfer function is the multipath model proposed in [16], [17], and [18]. This model proposed a top-down approach (statistical models), various parameters need to be determined through measurement. Statistical models do not require knowledge of either the link topology or the cable models, but they require an extensive measurement campaign.

An alternative model [19]-[20], based on transmission line theory, applies chain parameter matrices to describe by twoport network the relation between input and output voltage and current. This bottom-up model (deterministic models) is based on the knowledge of network topology and the cables' characteristic. Deterministic models require a detailed knowledge of the link topology and the cable models, but they do not require measurements.

It has been recently proposed that combining these two approaches to give rise to a hybrid model may indeed offer advantages [21]. In a hybrid model, a set of topologies must be defined and can be considered as a representative of the majority of topologies that can be found in real power line network. A specific scenario of topologies can be generated randomly or is based on a statistically relevant transfer function. Therefore, this article focuses on a hybrid model based on input measured transmission line parameters, set of topologies, and random generation of topology parameters.

\section{MODELS FOR INDOOR POWER LINE CHANNELS}

Indoor power line channel characteristic exhibits variation with time at various levels:

- There exists a long-time variation due to the switching of electrical appliances, which is related to human activity and, therefore, at random. 
- There appears a short-time variation as a result of the dependence on the mains voltage.

Based on these arguments, different timescales must be considered for modelling [21], [22]:

Random scale: This scale is related to the connection and disconnection of electric appliances. The change is random in time; it has no regular discretization in time, since the change is associated with the human activity in homes. It represents long-time variation and thus, for modelling the PLC a linear periodically time-variant system (LPTV) must be considered.

Periodic scale: This scale exhibits periodical variation synchronized with the cycle of the mains voltage. The channel is modelled as an LPTV system. The time unit is the mains period, $\mathrm{T}=20 \mathrm{~ms}(50 \mathrm{~Hz})$. The mains period can be divided into a series of shorter invariance intervals.

Invariant scale: At this level, the channel parameters are considered to be invariant. The linear time-invariant system (LTI) is adequate for modelling. The time interval can be selected according to the channel coherence time.

\section{LTI model}

When non-linearities of appliance loads are neglected, impedance appliances loads do not vary and the noise is considered stationary, the indoor power line can be considered to be an LTI system. For example, this assumption has been used in [18]. The LTI model is considered due to its simplicity. The LTI model can be preferable under several conditions, for example when the channel variation is not very important or the PLC is not capable of adapting to time variations.

The modelling of an entire power line network is difficult due to a large number of electrical appliances (even with the same features, but from different manufacturer). The measurement results in [23] showed the basic features of these appliances loads, in particular impedance is in most cases frequency-selective and shows resonant circuit shape. This assumption is proven by our measurement using the Bode 100 analyser. The results of impedance measurements are shown in Fig.1.

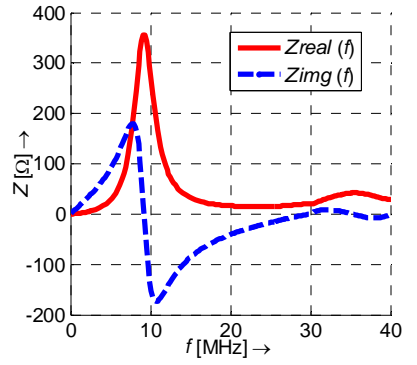

a)

Fig.1. Load impedance measurement. a) capacitive element of PLC modem (MCB10.2) b) inductive element of PLC modem (ICU-438).

\section{EXPERIMENTAL DISTRIBUTION NETWORK TOPOLOGY}

We have designed a distribution bus topology for the simulation issue. The topology structure is defined from averages of characteristic values of a typical structure of indoor PLC networks. The designed distribution topology and characteristic values of typical indoor PLC network were discussed in [24]. The proposal topology with four branches is shown in Fig.2. and Fig.3.

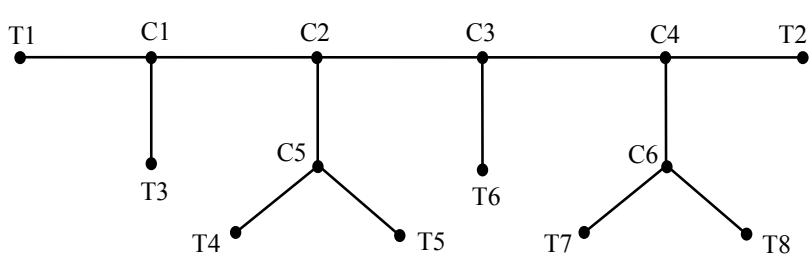

Fig.2. Simplified distribution network topology with four branches.

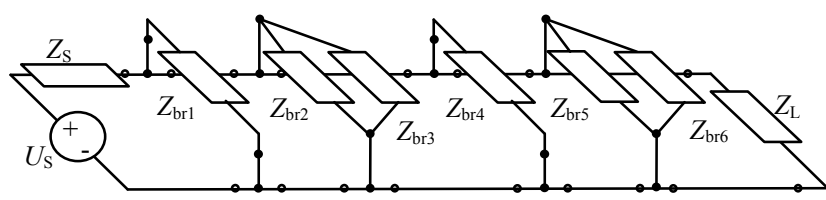

Fig.3. Distribution network topology with four branches.

\section{A. Two-port network model}

The method for modelling the transfer function of a power line channel uses the chain parameter matrices describing the relation between input and output voltage and current of two-port network (bottom-up model, see chapter 2).

The branches from topology in Fig.3. were replaced with equivalent impedance. The computation of equivalent impedance is described in [24]. The two-port network model is described in [24] and [25]. The simplified power line is shown in Fig.4. The entire line is composed of ten cascade matrices $\mathbf{A}_{i}$.

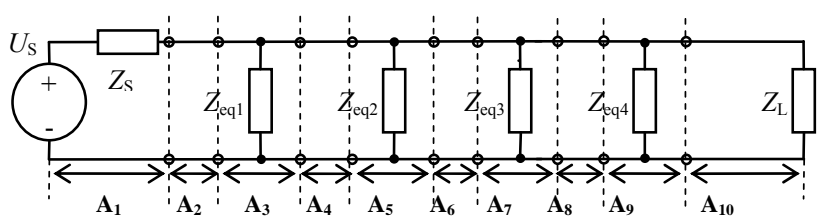

Fig.4. Simplified topology with four branches.

The resulting cascade matrix $\mathbf{A}$ of entire line (1) is obtained by multiplying cascade matrices $\mathbf{A}_{i}$. By putting particular elements of cascade matrix into (2), the transfer function of power line was obtained.

$$
\left[\begin{array}{c}
U_{1} \\
I_{1}
\end{array}\right]=\left[\begin{array}{ll}
A & B \\
C & D
\end{array}\right]\left[\begin{array}{l}
U_{2} \\
I_{2}
\end{array}\right]=\mathbf{A}\left[\begin{array}{c}
U_{2} \\
I_{2}
\end{array}\right]
$$

$$
H=\frac{U_{\mathrm{L}}}{U_{\mathrm{S}}}=\frac{Z_{\mathrm{L}}}{A Z_{\mathrm{L}}+B+C Z_{\mathrm{L}} Z_{\mathrm{S}}+D Z_{\mathrm{S}}}
$$




\section{B. Verification of two-port network power line model on simple experimental topology}

This chapter describes a comparison of the model with measurement. The comparison was verified on simple experimental topology with known parameters.

Fig.5. shows the experimental topology for the comparison of simulation results with measurement. Points $\mathrm{A}$ and $\mathrm{C}$ represent transmitter and receiver. Point D represents open bridge tap.

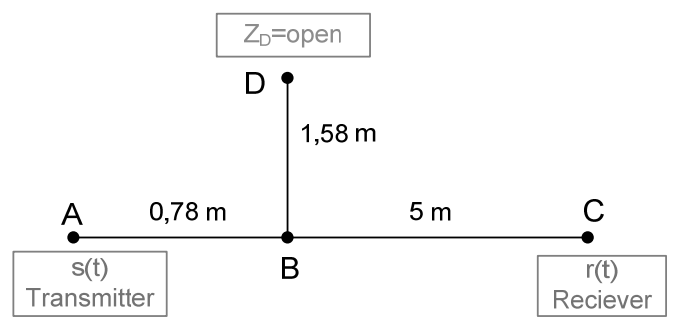

Fig.5. Experimental topology.

Frequency and phase response were measured by spectral analyser Instek GSP-830. Fig.6. shows the comparison with two-port network model. From the comparison, the agreement between measurement and simulation is obvious. The agreement shows the practicability and efficiency of the model.
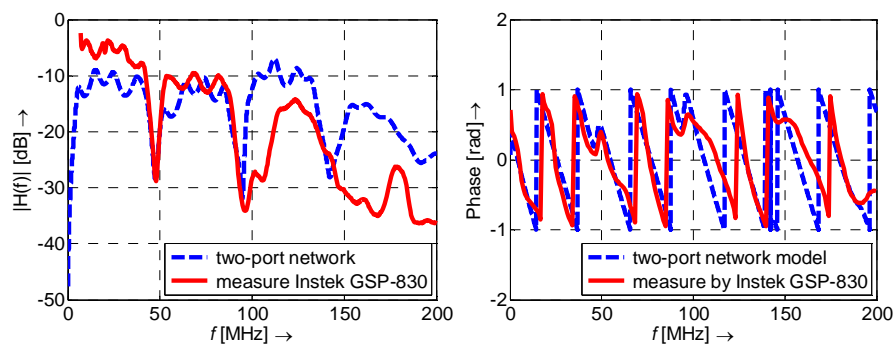

Fig.6. The comparison of frequency and phase response.

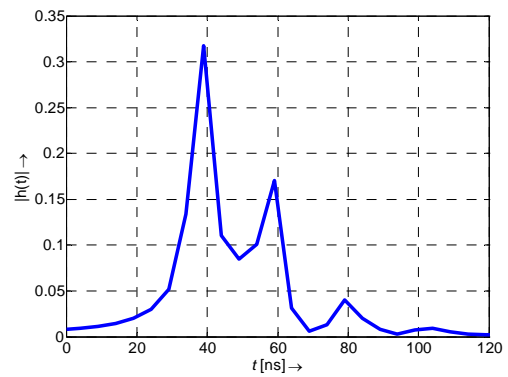

Fig.7. Impulse response from simulation.

The numerical analysis validated the comparison results too. Due to the insulating material with a dielectric constant $\varepsilon_{r} \approx 4$, the phase speed on the cable is approximately 150 $\mathrm{m} / \mu \mathrm{s}$. The first peak in Fig.7. is the transmission impulse from transmitter, which has traversed the entire cable length of $5.78 \mathrm{~m}$, thus appearing after time $5.78 / 150 \cdot 10^{6}=38.5 \mathrm{~ns}$. The second peak forms from the fact that the signal entering the tap and reflected at the tap's open end traversed the total length of $8.94 \mathrm{~m}(\mathrm{AB}+\mathrm{BD}+\mathrm{DB}+\mathrm{BC})$ appearing after $8.94 / 150 \cdot 10^{6}=59.6 \mathrm{~ns}$. The tap passage of $3.16 \mathrm{~m}$ takes about $21.1 \mathrm{~ns}$.

The effects of reflection in tap appear in the transfer function (frequency response) in the form of notches with fixed frequency spacing (see Fig.6.). The first notch occurs where the direct and the reflected waves are shifted exactly a half wavelength against each other, which leads to subtraction. The first frequency $f_{1}=47.4 \mathrm{MHz}$ belongs to the first notch, that means we have a period of $21.1 \mathrm{~ns}$. The repetition of notches occurs multiples of first frequency $f_{1}$.

\section{Model validation}

The most important characteristics of power line channel are attenuation and coherence bandwidth.

The attenuation of channel model is calculated by mean amplitude (in $\mathrm{dB}$ ) of their frequency response.

Coherence bandwidth is a direct measure of the channel frequency selectivity. The coherence bandwidth is the interval of frequencies in which the normalized autocorrelation function (see (3)) of the channel frequency response is higher than a certain value (usually set to 0.9 ). Or the coherence bandwidth is bandwidth in which the channel can be considered approximately flat [26].

$$
R(t, \Delta f)=\sum_{t=0}^{M} H(t, f) \cdot H(t, f+\Delta f)
$$

\section{INDOOR POWER LINE MODEL BASED ON RANDOM PARAMETERS}

A small number of sample models that represent the expected behaviour of typical network are more sufficient and more effective than replicating a multitude of particular power line networks. A possible way to achieve sample models is to generate randomly network topologies with well-chosen distribution parameters, and then solve the channel response by means of two-port network model (see chapter 4.A or [24]). These aspects are important for the model:

1. Parameters of power line cables. It is necessary to adopt the primary line parameter resistance $R^{\prime}(f)$, inductance $L^{\prime}(f)$, capacitance $C^{\prime}(f)$, conductance $G^{\prime}(f)$ and secondary line parameter characteristic impedance $Z_{\mathrm{C}}$ and propagation constant $\gamma$. These parameters are estimated according to cable characteristic from manufactures or obtained by measurement.

2. Topology layout. The topology of indoor power line network consists of branches (network sections), which are connected to a main path between the transmitter and receiver. Therefore, the parameters that need to be defined are: number of section, their length and relative position. These parameters will be obtained randomly.

3. Device characteristic. Individual branches represent devices (appliances) with the appropriate load impedances. The impedances can be obtained randomly or based on measurement.

Based on these aspects, the measurement of transmission parameters of power line cables was performed and the LTI power line generator was proposed. 


\section{A. Measurement of power line cable transmission parameters}

The measurements were carried out with a HP analyser 4192A, operating in the frequency range $5 \mathrm{~Hz}-13 \mathrm{MHz}$. The frequency field investigated was from $10 \mathrm{kHz}$ to 10 $\mathrm{MHz}$ according to the EN 50065-1 [27].

Cables CYKY, CYKYLo, and AYKY of different sizes were examined [29]. For the following modelling the cable CYKY $3 \times 2.5$ is considered, because these cables have the widest use in power engineering.

Fig.8. shows the measurements of the longitudinal parameters $R^{\prime}(f)$ and $L^{\prime}(f)$, and cross parameters $C^{\prime}(f)$ and $G^{\prime}(f)$ of cables CYKY.
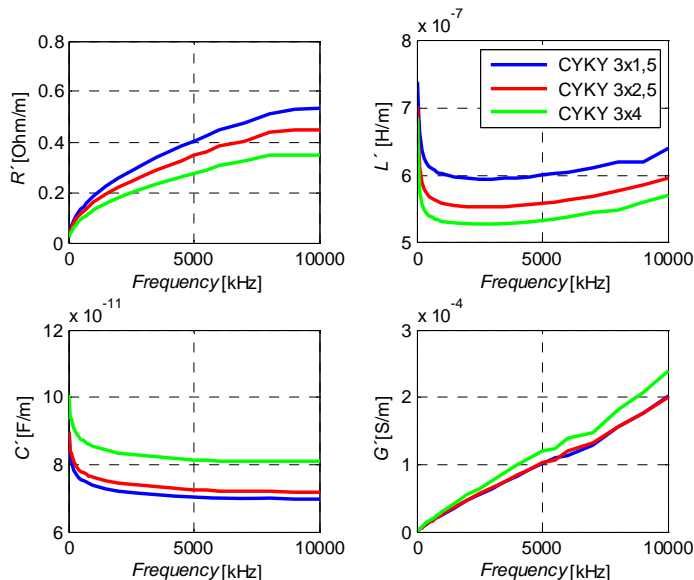

Fig.8. Measurement values of longitudinal parameters $R^{\prime}(f)$ and $L^{\prime}(f)$, and cross parameters $C^{\prime}(f)$ and $G^{\prime}(f)$.
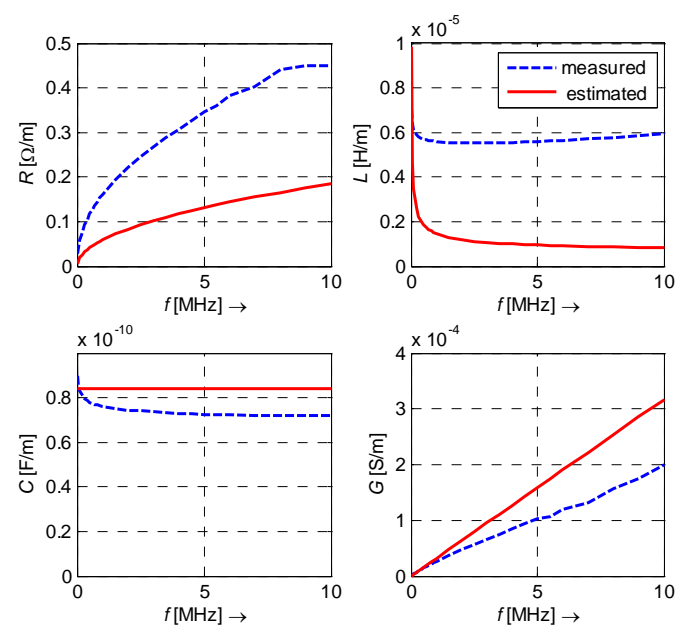

Fig.9. Comparison of transmission line parameters for CYKY $3 \times 2.5$ cable.

Fig.9. shows the comparison of measured and estimated transmission line parameters for the CYKY $3 \times 2.5$ cable. The estimated transmission line parameters are computed according to the [28] for CYKY $3 \times 2.5$ cable [29]. From the comparison it is evident that the evaluated cross parameter $C^{\prime}$ is not dependent on frequency, therefore it is constant in the whole range. The calculation of cross parameter $G^{\prime}(f)$ is based on the constant cross parameter $C^{\prime}(f)$, therefore it is often ignored in practice.
The most important transmission parameters that influence transmission over power line are the longitudinal parameters $R^{\prime}(f)$ and $L^{\prime}(f)$. The differences between measured and estimated values in Fig.9. may be caused by inaccurate values of cable parameters. The values of cable parameters are obtained from the manufacturer catalogue. These parameters had only an informative character, therefore did not match reality.

The most important parameters of transmission line are the secondary parameters $Z_{\mathrm{C}}$ and $\gamma$. The real part of propagation constant $\gamma$ corresponds to the attenuation constant $\alpha$. More information about primary and secondary parameters can be found in [30] and [31].

There are two different approaches to calculating the secondary parameters describing a transmission line. First, measuring the values of primary parameters $R^{\prime}(f), L^{\prime}(f)$, $C^{\prime}(f)$, and $G^{\prime}(f)$ to obtain the $Z_{C}$ and $\gamma$ parameters. Second, estimate the $Z_{C}$ and $\gamma$ parameters, taking into account the geometrical dimensions of the cable [29].

Fig.10. shows the magnitude of impedance $Z_{C}$ and the attenuation constant $\alpha$ measured and estimated in the frequency range $10 \mathrm{kHz}-10 \mathrm{MHz}$ for the CYKY $3 \times 2.5$ cable.
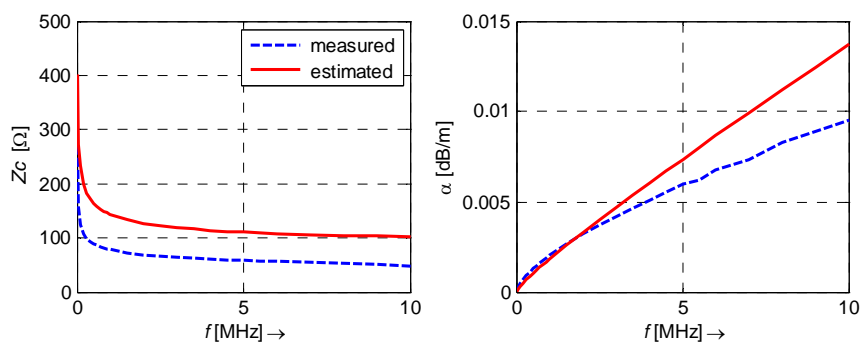

Fig.10. Magnitude of impedance $Z_{C}$ and the attenuation constant $\alpha$, measured and estimated for CYKY $3 \times 2.5$ cable.

It can be observed that there is good agreement between measured and estimated values of the $Z_{C}$ and $\alpha$. A comparison shows that the magnitude characteristic impedance and the attenuation constant are slightly higher for estimated values.

\section{B. LTI power line generator}

The LTI generator is based on the two-port network model. The proposed LTI generator contains these four main ideas:

1. The type of cable: In the generator, the cable type can be selected manually or randomly from:

- CYKY diameter $3 \times 1.5 ; 3 \times 2.5 ; 3 \times 4$,

- CYKYLo diameter 3x1.5;3x2.5,

- AYKY diameter 4x6; 4x10.

Based on cable type selection the primary and secondary parameters of power line cables are calculated.

2. Topology layout: As has been mentioned before, the parameters that need to be defined for topology layout are the number of sections, their length and relative position.

Based on [21], the average values of number of sections, the length of typical indoor PLC network are provided in Table 1 . In the proposed LTI generator, the parameters 
are chosen randomly with uniform distribution that is leading to random network realization.

The random sections are chosen by omitting randomly some of cascade matrix $\mathbf{A}_{i}$ from topology in Fig.4.

Table 1. Values to generate random topologies.

\begin{tabular}{|c|c|c|}
\hline $\begin{array}{c}\text { Topology scenario } \\
\left(\text { area, } \mathrm{m}^{2}\right)\end{array}$ & $\begin{array}{c}\text { Number of } \\
\text { sections }\end{array}$ & $\begin{array}{c}\text { Section length } \\
(\mathrm{m})\end{array}$ \\
\hline Small (60) & $5-6$ & $4-6$ \\
Medium (100) & $7-8$ & $5-10$ \\
Large (200) & $9-10$ & $10-20$ \\
\hline
\end{tabular}

3. Loads: Each load in the topology scenario can be selected among these four types of impedances manually or randomly:

- The first loading case $\mathbf{Z}_{1}$ regards the remaining points as being unloaded (open circuits).

- The second case $\mathbf{Z}_{2}$ considers different impedances connected to each terminal point. They correspond to the case of: low impedance, RF impedance, similar to cable characteristic $Z_{C}$, high impedance and open circuit $\left(\mathbf{Z}_{2}=\left[5,50,75,1000,1 \cdot 10^{8}\right] \Omega\right)$.

- The third case $\mathbf{Z}_{3}$ assumes that constant value impedance is connected to every termination.

- The fourth case, frequency selective function, the impedance values can be defined as the impedance of parallel RLC resonant circuit that contains these parameters: $R$, resistance at resonance; $\omega_{0}$ resonance angular frequency; $Q$, quality factor, and it is defined by the equation:

$$
Z(\omega)=\frac{R}{1+\mathrm{j} Q\left(\frac{\omega}{\omega_{0}}-\frac{\omega_{0}}{\omega}\right)}
$$

Based on [21] the appropriate values for the frequency selective function are $R \in(200,1800) \Omega$; $Q \epsilon(5.25)$ and $\omega_{0} / 2 \pi \epsilon(2.28) \mathrm{MHz}$.

\section{TRANSFER FUNCTION ANALYSIS AND SIMULATION RESULTS - LTI GENERATOR}

\section{A. Simulation results for loads with different impedances}

In Fig.11. the module and phase of the transfer function generated randomly with LTI generator with these randomly generated parameters is shown:

- CYKY diameter 3x2.5.

- Medium topology, number of sections: 7, omitted section 2, 4, 7 .

- $\quad$ Load impedances $\mathbf{Z}$ in $[\Omega]=\left[Z_{b r 1}, Z_{b r 2}, Z_{b r 3}, Z_{b r 4}, Z_{b r 5}\right.$, $\left.Z_{b r 6}\right]=\left[50,1 \cdot 10^{8}, 50,1000,75,75\right]$.

- Section lengths in [m]: $\left[l t_{11}, l c_{21}, l c_{32}, l c_{43}, l t_{24}, d_{\mathrm{br} 1}, d_{\mathrm{br} 2}\right.$, $\left.d_{\mathrm{br} 3}, d_{\mathrm{br} 4}, d_{\mathrm{br} 5}, d_{\mathrm{br} 6}\right]=[6,7,9,9,6,6,8,8,7,6,10,5$, 6], where $l t_{i j}$ - length between $\mathrm{T}_{i}$ and $\mathrm{C}_{j}, l c_{i j}$ - length between $\mathrm{C}_{i}$ and $\mathrm{C}_{j}$ (see Fig.2.).

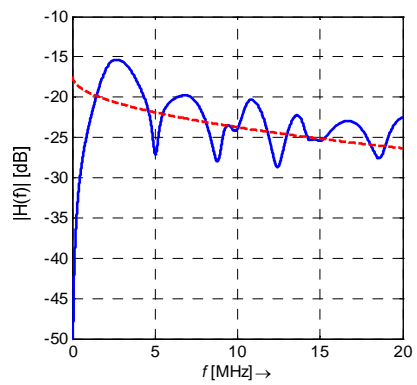

a)

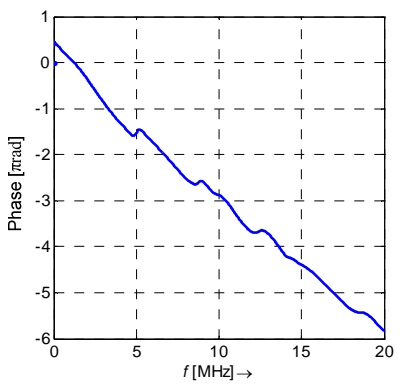

b)
Fig.11. Transfer function of medium topology with different impedances: a) frequency response b) phase response.

Results for medium topology with 7 sections:

- The average mean attenuation of topology with 7 sections is $-23.01 \mathrm{~dB}$.

- Coherence bandwidth set with the 0.9 level according to (3) is $964 \mathrm{kHz}$.

Red waveform in Fig.11. represents a module frequency response interleaving and it is obtained by attenuation equation:

$$
|H(f)|=a \sqrt{f}+b,
$$

where $a$ and $b$ parameters are obtained based on the method of least squares.

\section{B. Comparison of all topologies with constant load impedances}

Fig.12. shows the comparison of transfer functions for all three topologies obtained by the LTI generator. It shows attenuation increase with increasing of the topology size.

The average attenuation for large topology is $-31.74 \mathrm{~dB}$ and for small topology only $-14.78 \mathrm{~dB}$. Additional bridge taps (large topology) lead to worse channel results.

Table 2. shows the coherence bandwidth set with the 0.9 level according to (3) for all three topologies obtained by LTI generator.



a)

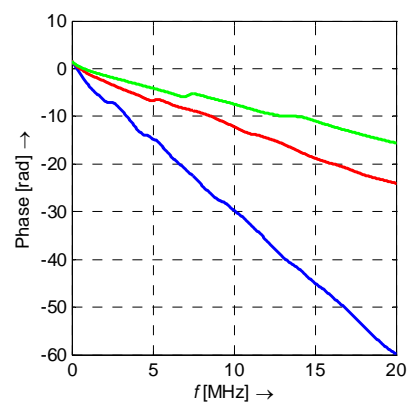

b)
Fig.12. Transfer function comparison for all topologies with constant load impedances: a) frequency response b) phase response. 
Table 2. Coherence bandwidth for topologies with constant load impedances.

\begin{tabular}{|c|c|}
\hline $\begin{array}{c}\text { Topology scenario } \\
\left(\text { area, } \mathrm{m}^{2}\right)\end{array}$ & $\begin{array}{c}\text { Coherence } \\
\text { bandwidth }[\mathrm{kHz}]\end{array}$ \\
\hline Small (60) & 1341 \\
Medium (100) & 995.5 \\
Large (200) & 415 \\
\hline
\end{tabular}

\section{Simulation results for frequency selective load impedances}

In Fig.13. the module and phase of the transfer function randomly generated with LTI generator with these randomly generated parameters is shown:

- CYKY diameter 3x2.5.

- Large topology, number of section: 10.

- Load impedances: RLC resonant circuit (frequency selective function) is shown in Fig.14.

- Section lengths in [m]: $\left[l t_{11}, l c_{21}, l c_{32}, l c_{43}, l t_{24}, d_{b r r}\right.$, $\left.d_{b r}, d_{b r 3}, d_{b r}, d_{b r}, d_{b r 6}\right]=[12,14,19,14,19,14,17$, $16,11,14,19,11,14$ ].

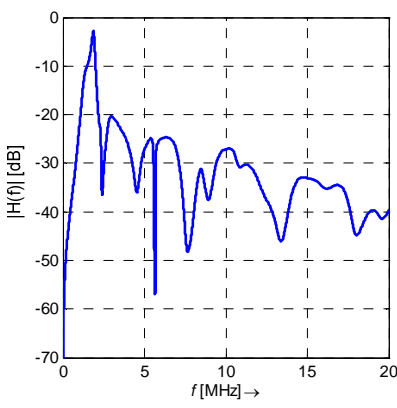

a)

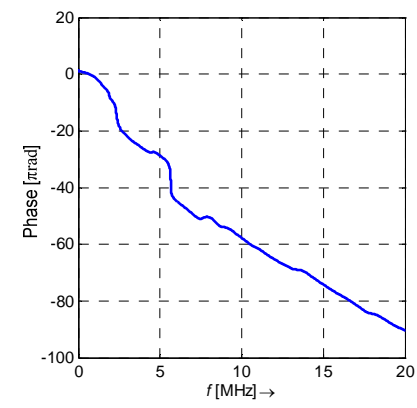

b)
Fig.13. Transfer function for large topology with frequency selective impedances: a) frequency response b) phase response.

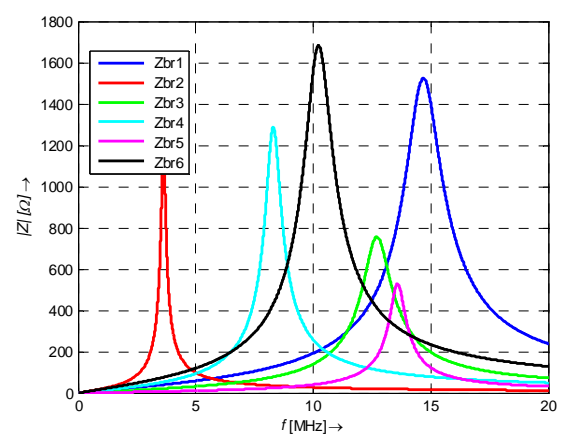

Fig.14. Frequency selective function of load impedances.

Results for large topology with 10 sections:

- The average mean attenuation of topology with 10 sections is $-32.54 \mathrm{~dB}$.

- Coherence bandwidth set with the 0.9 level according to (3) is $404.5 \mathrm{kHz}$.

\section{Comparison of all topologies with frequency selective impedances}

Fig.15. shows the comparison of transfer functions for all three topologies obtained by the LTI generator.

The average attenuation for large topology is $-32.54 \mathrm{~dB}$, for medium topology is $-22.30 \mathrm{~dB}$ and for small topology only $-15.36 \mathrm{~dB}$.

Table 3. shows the coherence bandwidth set with the 0.9 level according to (3) for all three topologies obtained by LTI generator.

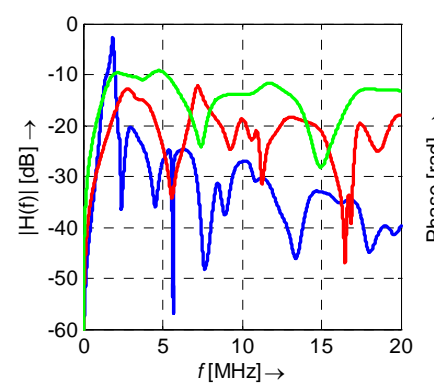

a)

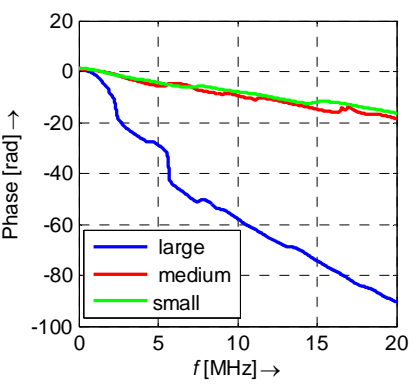

b)
Fig.15. Transfer function comparison for all topologies with frequency selective impedances: a) frequency response b) phase response.

Table 3. Coherence bandwidth for topologies with frequency selective impedances.

\begin{tabular}{|c|c|}
\hline $\begin{array}{c}\text { Topology scenario } \\
\left(\text { area, } \mathrm{m}^{2}\right)\end{array}$ & $\begin{array}{c}\text { Coherence } \\
\text { bandwidth }[\mathrm{kHz}]\end{array}$ \\
\hline Small (60) & 1051.5 \\
Medium (100) & 584 \\
Large (200) & 404.5 \\
\hline
\end{tabular}

\section{CONCLUSION}

The methodology presented can be used to describe the channel transmission behaviour in terms of network physical structure and loading conditions.

The generator created allows generating LTI channel responses. The aim of the LTI power line channel generator is not to exactly reproduce the characteristic of a given power line network, but to obtain an expectable channel response which can be used for testing new transmission techniques. The generator parameters have been proposed according to the network configurations, while different parameter values can be chosen to create channels with different characteristics.

The most important characteristics of an LTI channel are the attenuation and the frequency selection (evaluated by means of coherence bandwidth). In case of constant load impedances, the average attenuation for large topology with four branches was $-31.74 \mathrm{~dB}$ and for small topology only $14.78 \mathrm{~dB}$. Coherence bandwidth for large topology was $415 \mathrm{kHz}$ and for small topology $1341 \mathrm{kHz}$. A reduction of the number of bridge taps (small topology) leads to a better channel with lower attenuation. 
In case of frequency selective impedances, the average attenuation and coherence bandwidth were the worst for all topologies. The frequency selective impedances lead to the worst channel with higher attenuation.

As the loading conditions change, the channel may experience deep fading conditions that make some subchannels unsuitable for data transmission. Therefore, the proposed generator in this article can be helpful in evaluating the performance of power line channels.

\section{ACKNOWLEDGMENT}

This research work is funded by the Technology Agency of Czech Republic project No. TA02020856.

Measurements were run on computational facilities of the SIX Research Center, the registration number CZ.1.05/2.1.00/03.0072.

\section{REFERENCES}

[1] Gellings, C.W. (2009). The Smart Grid: Enabling Energy Efficiency and Demand Response. CRC Press.

[2] Trulove, J.G. (2003). The Smart House. Collins Design.

[3] Oksa, P., Soini, M., Sydanheimo, L., Kivikoski, M. (2006). Considerations of using power line communication in the AMR system. In IEEE International Symposium on Power Line Communications and Its Applications. IEEE, 208-211.

[4] Havelka, J., Malarić, R., Frlan, K. (2012). Staged-fault testing of distance protection relay settings. Measurement Science Review, 12 (3), 111-120.

[5] Pang, Q., Liu, X., Sun, B., Ling, Q. (2012). Approximate entropy based fault localization and fault type recognition for non-solidly earthed network. Measurement Science Review, 12 (6), 213-313.

[6] Cortés, J., Díez, L., Cañete, F., Sánchez-Martínez, J. (2010). Analysis of the indoor broadband power-line noise scenario. IEEE Transactions on Electromagnetic Compatibility, 52 (4), 849-858.

[7] Galli, S. (2010). A simple two-tap statistical model for the power line channel. In IEEE International Symposium on Power Line Communications and its Applications (ISPLC), 28-31 March 2010. IEEE, 242-248.

[8] Hasirci, Z., Cavdar, I.H., Suljanović, N., Mujčić, A. (2013). An application of the broadband PLC for smart homes in Turkey. In 36th International Conference on Telecommunication and Signal Processing (TSP), 2-4 July 2013. Budapest: Asszisztencia Szervezo Kft., 222-226.

[9] International Telecommunication Union. G.9960 : Unified high-speed wire-line based home networking transceivers - system architecture and physical layer specification. www.itu.int/rec/T-REC-G.9960/en.

[10] Roka, R. (2012). Fixed transmission media. In Technology and Engineering Applications of Simulink. Rijeka, Croatia: InTech, 27.
[11] Mlynek, P., Misurec, J., Koutny, M. (2012). Noise modeling for power line communication model. In 35th International Conference on Telecommunications and Signal Processing (TSP), 3-4 July 2012. IEEE, 282-286.

[12] Mlynek, P., Misurec, J., Koutny, M., Slavicek, K. (2012). Modelling of part medium access methods in the HomePlug. Przeglad Elektrotechniczny, 1, 91-94.

[13] Mlynek, P., Koutny, M., Misurec, J. (2010). Model of power line communication system. In 33rd International Conference on Telecommunication and Signal Processing (TSP), 17-20 August 2010. Budapest: Asszisztencia Szervezo Kft., 406-410.

[14] Galli, S., Scaglione, A., Wang, Z. (2011). For the grid and through the grid: The role of power line communications in the smart grid. Proceedings of the IEEE, 99 (6), 998-1027.

[15] Bestak, I., Orgon, M. (2011). Performance measurement of encryption algorithms used in PLC devices. International Journal of Research and Reviews in Computer Science, 2 (5), 1218-1221.

[16] Philipps, H. (1999). Modeling of power line communication channels. In Int. Symposium on Power Line Communications and Its Applications (ISPLC), 30 March - 1 April 1999. Shannon Foundation, 14-21.

[17] Dostert, K. (2005). Propagation channel characterization and modeling: Outdoor power supply grids as communication channels. In International Symposium on Power Line Communications and Its Applications (ISPLC), 6-8 April 2005. IEEE.

[18] Zimmermann, M., Dostert, K. (2002). A multipath model for the powerline channel. IEEE Transactions on Communications, 50 (4), 553-559.

[19] Esmailian, T., Kschischang, F., Gulak, G. (2002). An in-building power line channel simulator. In International Symposium on Power Line Communications and Its Applications (ISPLC), 27-29 March 2002. IEEE, 1-5.

[20] Galli, S., Banwell, A. (2005). A novel approach to accurate modeling of the indoor power line channelPart II: Transfer function and channel properties. IEEE Transactions on Power Delivery, 20 (3), 1869-1878.

[21] Ferreira, H., Lampe, L., Newbury, J., Swart, T.G. (2010). Power Line Communications: Theory and Applications for Narrowband and Broadband Over Power Lines. John Wiley.

[22] Sancha, S., Canete, J.F., Diez, L., Entrambasaguas, T. (2007). A channel simulator for indoor power-line communications. In IEEE International Symposium on Power Line Communications and Its Applications (ISPLC '07), 26-28 March 2007. IEEE, 104-109.

[23] Corripio, F.J.C., Arrabal, J.A.C., del Rio, L.D., Munoz, J.T.E. (2006). Analysis of the cyclic shortterm variation of indoor power-line channels. IEEE Journal on Selected Areas in Communications, 24 (7), 1327-1338.

[24] Mlynek, P., Misurec, J., Koutny, M., Silhavy, P. (2012). Two-port network transfer function for power line topology modeling. Radioengineering, 21 (1), 356-363. 
[25] Mlynek, P., Koutny, M., Misurec, J. (2011). Power line cable transfer function for modelling of power line communication systems. Journal of Electrical Engineering, 2, 1-4.

[26] Proakis, J.G. (2001). Digital Communications, 4th ed. McGraw-Hill.

[27] CENELEC. (2008). Signalling on low-voltage electrical installations in the frequency range $3 \mathrm{kHz}$ to $148.5 \mathrm{kHz}$. EN50065-1.

[28] Papaleonidopoulos, I., Karagiannopoulos, C., Theodorou, N., Anagnostopoulos, C.,

Anagnostopoulos, I. (2002). Modelling of indoor low voltage power-line cables in the high frequency range. In International Symposium on Power Line Communications and Its Applications (ISPLC), 27-29 March 2002. IEEE.
[29] Draka kabely, s.r.o. (2007). Power cables and flexible cables. www.draka.cz/images/stories/katalog/silove kabely/1-silove-cz-web.pdf.

[30] Cataliotti, A., Daidone, A., Tine, G. (2008). Power line communication in medium voltage systems: Characterization of MV cables. IEEE Transactions on Power Delivery, 23 (4), 1896-1902.

[31] Roka, R. (2012). Verification of characteristics of the parametric model for the RC4 reference channel. In 35th International Conference on Telecommunications and Signal Processing (TSP), 3-4 July 2012. IEEE, 136-140.

Received February 6, 2013 Accepted July 30, 2013. 